Ann. Zootech., I974, 23 (2), II9-I32.

\author{
I.N.R.A. \\ BIBLIOTHEQUE UÓ 35906 \\ Domaine de CROUELLE \\ 63039 \\ CLERMONT-FD CEBEX 2 \\ 712102
}

\title{
SUPPLÉMENTATION EN LYSINE D'UN RÉGIME A BASE DE TOURTEAU DE SÉSAME CHEZ LE LAPIN. EFFETS SUR LES PERFORMANCES DE CROISSANCE ET LE BILAN AZOTÉ ESTIMÉ PAR DEUX MÉTHODES
}

\author{
M. COLIN \\ avec la collaboration technique de G. SARdi DE LETro \\ Station de Recherches sur l'Élevage des Porcs, \\ Centre national de Recherches zootechniques, I. N. R. A., \\ 78350 Jouy en Josas
}

\section{RÉSSUMÉ}

L'étude chez le Lapin des effets de la supplémentation de régimes à base de tourteau de sésame par la L-lysine a été réalisée suivant un schéma factoriel comprenant 3 taux de matières azotées (I 3-17-2I p. roo) et 3 doses de lysine additionnelle (o-1,5-3 p. roo des matières azotées).

La supplémentation en lysine améliore les performances de croissance. Ces effets sont particulièrement marqués à $\mathrm{I} 7 \mathrm{p}$. Ioo de matières azotées. Ils sont moins importants à $\mathrm{I} 3 \mathrm{p}$. roo de matières azotées et les gains de poiđs obtenus restent médiocres. Enfin, ils sont faibles à 2 I p. IOO de matières azotées.

Ces résultats permettent d'annoncer pour le besoin en matières azotées et le besoin en lysine des valeurs de l'ordre de I 7 p. I oo et $0,65 \mathrm{p}$. Ioo du régime.

En outre, une étude en cages à métabolisme pour 5 des régimes précédents (les 3 régimes non supplémentés et les 2 régimes à 17 p. I oo de matières azotées supplémentées) montre que :

- le bilan azoté évolue de façon parallèle au gain de poids,

- l'élévation du taux de matières azotées abaisse le coefficient de rétention azotée et améliore l'utilisation digestive apparente de l'azote,

- la supplémentation en L-lysine augmente le coefficient de rétention azotée et ne modifie pas la digestibilité apparente des matières azotées.

Par ailleurs, à l'occasion de cette expérience, on a comparé les bilans azotés établis par collecte et par analyse corporelle. La différence enregistrée - de i I à I 9 p. Ioo - est de l'ordre de celles rapportées par la littérature pour d'autres espèces. Cependant, les 2 types de bilans permettent de classer de façon identique les régimes.

\section{INTRODUCTION}

GAMAN et FISHER (I97I) ont récemment démontré que l'addition de lysine à un régime déficient en cet acide aminé améliore les performances de croissance du Lapereau sevré. D'autres auteurs (CHEEKE, I97I) estiment le besoin en lysine 
du Lapin à environ 0,9 p. Ioo du régime. De plus, un premier essai (CoLrN, I973) a montré que la supplémentation en lysine d'un régime à base de tourteau de sésame, connu pour sa déficience en lysine, permettait une forte augmentation des performances pondérales des animaux.

Nous avons donc poursuivi ces travaux en étudiant les effets de la supplémentation à différents taux de matières azotées et en précisant son action sur 1'utilisation digestive et métabolique des matières azotées alimentaires.

\section{MATÉRIEL E'T MÉTHODES}

\section{Présentation des expériences}

Dans la première expérience, nous avons enregistré, pendant 5 semaines, les performances de croissance et de consommation obtenues avec 9 régimes correspondant à la combinaison factorielle de 3 taux de matières azotées (I 3-I 7-2I p. IOO) et de 3 taux de L-lysine additionnelle (0-I,53 p. Ioo des matières azotées).

Dans la seconde expérience, nous avons étudié pendant 3 semaines le bilan azoté obtenu avec 5 des régimes précédents : les 3 régimes non supplémentés et les 2 régimes à $17 \mathrm{p}$. Ioo de matières arotées supplémentées.

Nous avons déterminé le bilan azoté à la fois par collecte des excreta et par analyse corporelle ce qui a permis une comparaison des deux méthodes d'estimation.

\section{Animaux}

Les expériences ont été réalisées avec des lapereaux californiens des deux sexes, âgés de 6 semaines en début d'expérience. Les performances de croissance des animaux ont donc été étudiées pendant la majeure partie de la période d' " engraissement " comprise entre le sevrage (4 semaines) et l'abattage (I I- I 2 semaines).

Les 8I animaux de la première expérience étaient logés dans des cages individuelles dans les conditions déjà décrites (Colin, Arkhurst et LeBas, 1973). Les 25 animaux de la seconde expérience étaient placés en cages à métabolisme permettant la séparation et la collecte quantitative de l'urine et des fèces. Les deux types de cage étaient de dimension suffisante pour permettre une pratique de la coprophagie normale par les animaux.

\section{Régimes}

Les protéines des régimes étaient apportées presque exclusivement par le tourteau de sésame (tabl. I). Une faible quantité de glutamate de sodium dans les régimes non supplémentés a permis d'ajouter la L-lysine en substitution partielle du glutamate sans modifier ia teneur en azote du régime. Les aliments étaient présentés sous forme de granulés de $2,5 \mathrm{~mm}$ de diamètre.

\section{Contrôle des performances de croissance et de consommation}

Les animaux ont été pesés individuellement une fois par semaine vers 9 heures du matin. Les consommations individuelles ont été contrôlées 2 fois par semaine, par différence entre les quantités d'aliments distribuées et refusées.

\section{Détermination du bilan par la méthode des collectes}

Les animaux étaient placés individuellement dans des cages à métabolisme comprenant : - un chassis portant une mangeoire et un abreuvoir automatique. Ce chassis est entouré 
d'une tôle à la partie inférieure afin d'éviter les pertes d'urine par projection. Le lapin est placé sur une première grille à mailles rectangulaires $(20 \times$ ro $\mathrm{mm})$ de fil galvanisé permettant le passage des fèces. Celles-ci sont recueillies sur une seconde grille à mailles plus fines ;

- un bec en forme d'entonnoir recueille l'urine collectée sous acide sulfurique dans un bécher.

Les consommations sont enregistrées tous les jours. Urines et fèces sont recueillies quotidiennement, stockées à - I $5^{\circ} \mathrm{C}$ et homogénéisées en fin d'expérience. Un échantillon est alors prélevé pour analyses.

TABL,EAU I

Composition des régimes expérimentaux

\begin{tabular}{|c|c|c|c|c|c|c|c|c|c|}
\hline Matières azotées & \multicolumn{3}{|c|}{13} & \multicolumn{3}{|c|}{17} & \multicolumn{3}{|c|}{21} \\
\hline $\begin{array}{l}\text { L-lysine supplémentaire } \\
\text { p. } 100 \text { protéines }\end{array}$ & 0 & 1,5 & 3 & 0 & 1,5 & 3 & 0 & 1,5 & 3 \\
\hline Lot & 1 & 2 & 3 & 4 & 5 & 6 & 7 & 8 & 9 \\
\hline \multicolumn{10}{|l|}{ Composition } \\
\hline Tourteau de sésame $\left({ }^{1}\right) \ldots \ldots$ & 28,7 & 28,7 & 28,7 & 38,2 & 38,2 & 38,2 & 47,5 & 47,5 & 47,5 \\
\hline Amidon de maĩs $\ldots \ldots \ldots$ & 42,8 & 43,1 & 43,5 & 32,9 & 33,4 & 33,8 & 23,1 & 23,6 & 24,2 \\
\hline Huile d'arachide ... & 3 & 3 & 3 & 3 & 3 & 3 & 3 & 3 & 3 \\
\hline Paille de blé $\ldots \ldots \ldots \ldots$ & 20 & 20 & 20 & 20 & 20 & 20 & 20 & 20 & 20 \\
\hline C. M. V. $\left({ }^{2}\right) \ldots \ldots \ldots$ & 4 & 4 & 4 & 4 & 4 & 4 & 4 & 4 & 4 \\
\hline L-lysine $\mathrm{HCl}\left({ }^{3}\right)$ & - & 0,26 & 0,52 & $\ldots$ & 0,34 & 0,68 & - & 0,42 & 0,84 \\
\hline Glutamate de $\mathrm{Na} \ldots \ldots \ldots$ & 1,46 & 0,86 & 0,26 & 1,92 & 1,13 & 0,34 & 2,37 & 1,40 & 0,43 \\
\hline \multicolumn{10}{|l|}{ Résultats d'analyse } \\
\hline Matière sèche $\ldots \ldots \ldots \ldots$ & 92,1 & 90,9 & 90,9 & 93,1 & 92,3 & 90,8 & 91,7 & 91,2 & 91,6 \\
\hline Matière organique $\ldots \ldots \ldots$ & 83,0 & 83,4 & 84,3 & 82,3 & 81,8 & 81,1 & 79,7 & 80,2 & 79,8 \\
\hline Matières azotées $(\mathrm{N} \times 6,25)$ & 13,4 & 13,3 & 12,8 & 17,5 & 17,6 & 17,7 & 21,2 & 21,6 & 22,0 \\
\hline Cellulose $\ldots \ldots \ldots \ldots \ldots \ldots$ & 10,0 & $\left({ }^{4}\right)$ & (4) & 10,7 & 10,6 & 10,5 & 11,1 & $(\mathbf{4})$ & $\left({ }^{4}\right)$ \\
\hline $\begin{array}{r}\text { Teneur calculée en lysine } \\
\text { totale } \ldots \ldots \ldots \ldots \ldots \ldots \ldots\end{array}$ & 0,28 & 0,48 & 0,67 & 0,38 & 0,63 & 0,88 & 0,48 & 0,80 & 1,11 \\
\hline
\end{tabular}

(1) Taux protéique 39,7 p. 100 .

(2) Colin, ARkhurst et Lebas, 1973.

(3) Produit commercialisé par Rhône-Poulenc : 95 p. 100 de L-lysine $\mathrm{HCl}$ à 80 p. 100 de lysine base.

(4) Données non analysées.

\section{Détermination du bilan par analyse corporelle}

Les 25 animaux ont été sacrifiés à la fin de l'expérience par injection de $3 \mathrm{~cm}^{3}$ de Nembutal dans la veine marginale de l'oreille. Ils ont été aussitôt congelés, puis découpés et broyés une première fois dans un broyeur à lames Robot-Coupe, puis successivement dans un hâchoir à grilles de $8 \mathrm{~mm}$ et $3 \mathrm{~mm}$ de diamètre. L'échantillon obtenu a été ensuite lyophilisé, puis la lyophilisat rebroyé et homogénéisé dans un deuxième broyeur à lames Robot-Coupe. Un échantillon représentatif a alors été prélevé en vue d'analyses.

A fin de déterminer la quantité d'azote que contenaient les animaux en début d'expérience, 5 lapins, correspondant aux 5 répétitions ont été sacrifiés au commencement de l'étude et analysés dans les mêmes conditions que les précédents. 
M. COLIN

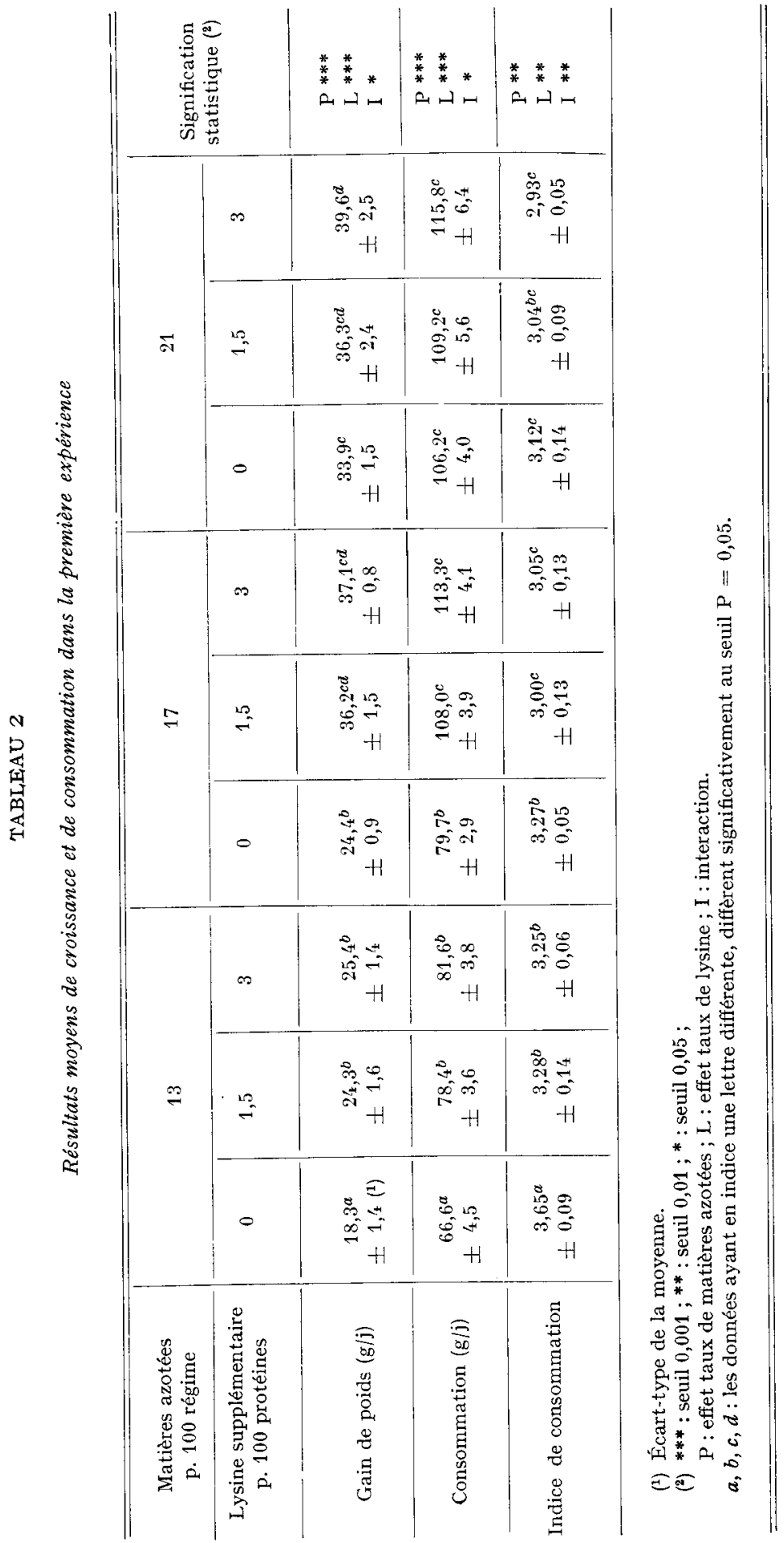




\section{RÉSULTATS}

\section{A. - Croissance et efficacité alimentaire}

Le gain de poids, la consommation et l'indice de consommation sont significativement améliorés par l'augmentation du taux protéique ainsi que par la supplémentation en lysine (tabl. 2). En outre, l'analyse statistique met en évidence une interaction significative entre le taux protéique et le niveau de supplémentation en lysine. Cela signifie que l'addition d'une même proportion de lysine entraîne des effets différents selon la teneur en matières azotées du régime : la supplémentation par I,5 p. Ioo de lysine améliore le gain de poids de $32 \mathrm{p}$. Ioo à I3 p. Ioo de matières azotées, 48 p. Ioo à 17 p. Ioo et de 9 p. Ioo à 21 p. IOO. Les différences enregistrées entre les résultats correspondant aux régimes à $\mathrm{I}, 5$ et 3 p. Ioo de lysine supplémentaire sont faibles.

L'augmentation du taux de protéines n'a pas les mêmes conséquences lorsque les régimes sont ou non additionnés de lysine. Ainsi, on observe pour les seuls régimes non supplémentés une amélioration significative des performances entre 17 et 2I p. roo de matières azotées.

En outre, le niveau des performances permet de diviser nos régimes en deux groupes (fig. I et 2). Ainsi, les croissances obtenues avec les 3 régimes à I3 p. Ioo de matières azotées et celui à $\mathrm{I} 7 \mathrm{p}$. Ioo non supplémenté sont relativement médiocres ; par contre celles enregistrées pour les 5 autres régimes sont d'un bon niveau et leurs valeurs sont comparables.

Toutefois, pour ces 5 régimes, le gain de poids augmente légèrement avec la quantité de lysine ingérée. Une corrélation étroite $(r=0,97)$ existe entre les variations de ces deux facteurs. Cette liaison s'exprime par l'équation de régression :

$$
\mathrm{Y}=6,65 \mathrm{X}+30,84
$$

où $\mathrm{Y}$ est le gain de poids en grammes par jour ;

$\mathrm{X}$ la quantité de lysine ingérée en grammes par jour.

\section{B. - Digestibilité}

On observe des différences significatives entre les coefficients d'utilisation digestive pour les 3 régimes non supplémentés. Or ceux-ci se distinguent à la fois par leur taux protéique et par leur teneur en cellulose. En effet, les protéines étant apportées principalement par le tourteau de sésame, leur augmentation dans la ration va de pair avec celle de la cellulose. Les résultats montrent que :

- les coefficients d'utilisation digestive apparente de la matière sèche, de la matière organique et de l'énergie décroissent lorsque le taux d'incorporation du tourteau de sésame s'élève;

- au contraire, dans ces conditions, les digestibilités apparentes des matières azotées et de la cellulose sont améliorées.

La supplémentation en lysine n'affecte aucun des coefficients d'utilisation digestive apparente mesurés. 


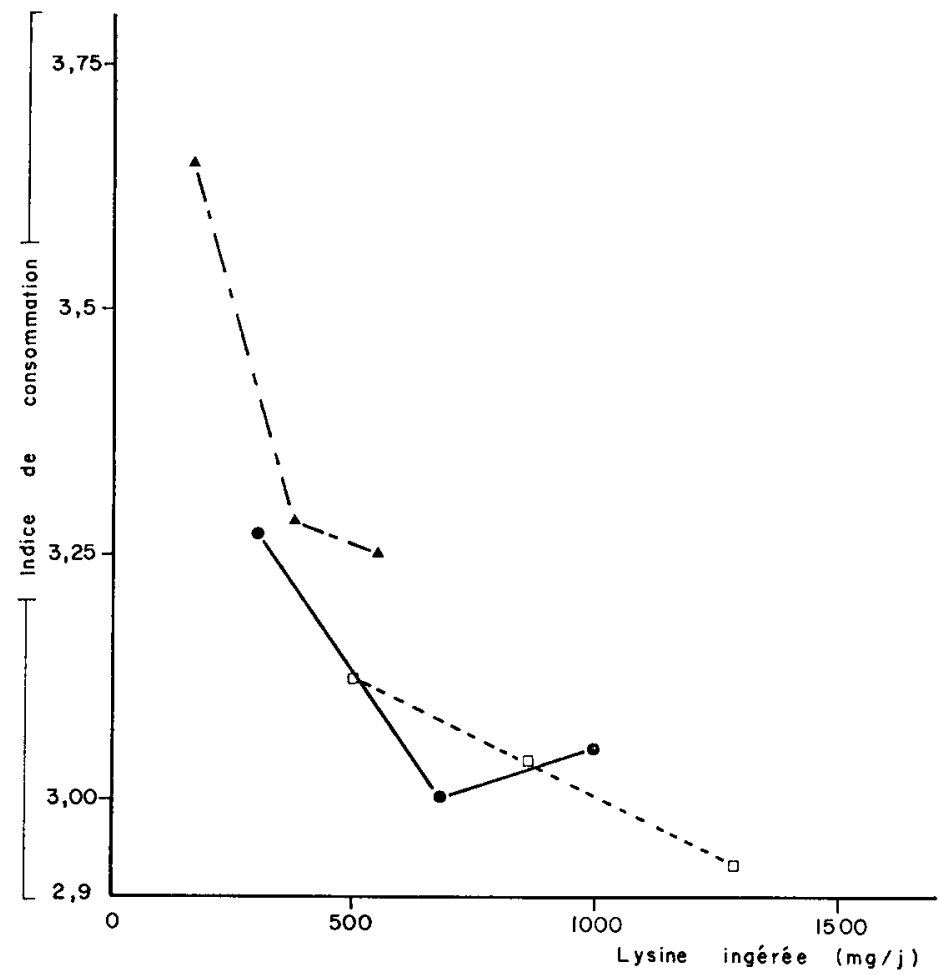

FIG. I. - Relation entre l'indice de consommation et la quantité de lysine ingérée

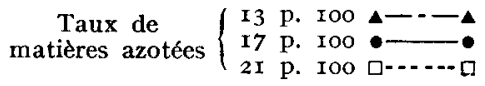

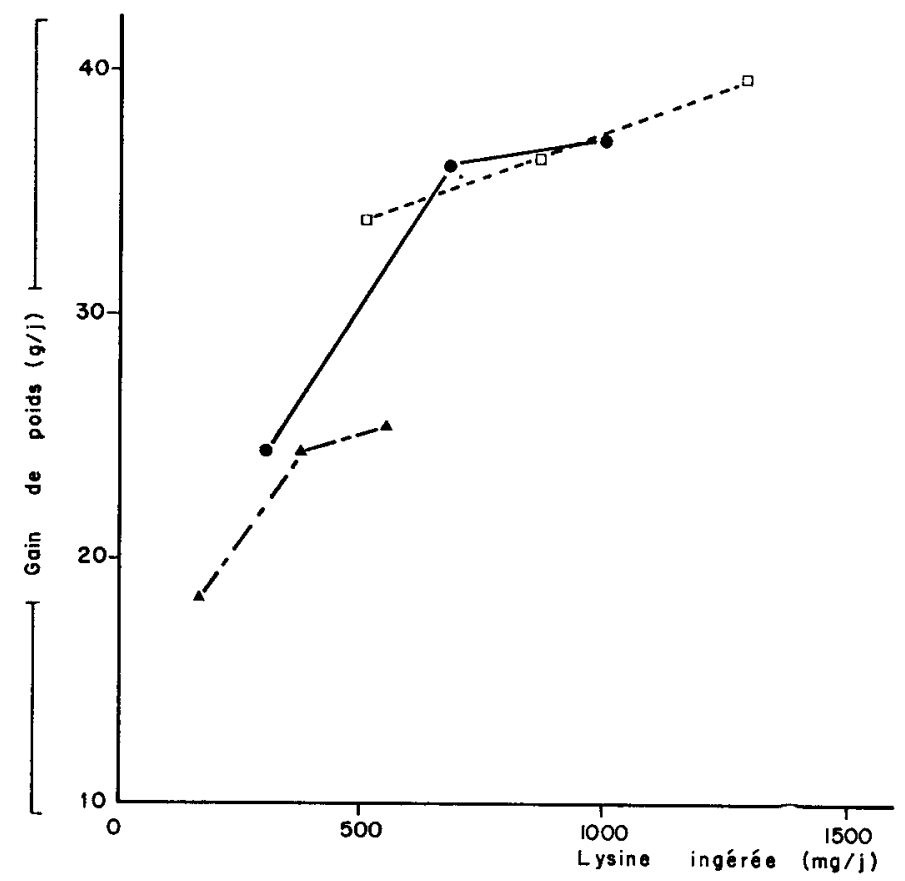

FIG. 2. - Relation entre le gain de poids et la quantité de lysine ingérée

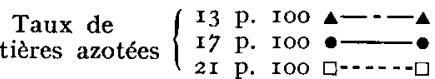


BESOIN EN LYYSINE DU IAAPIN EN CROISSANCE

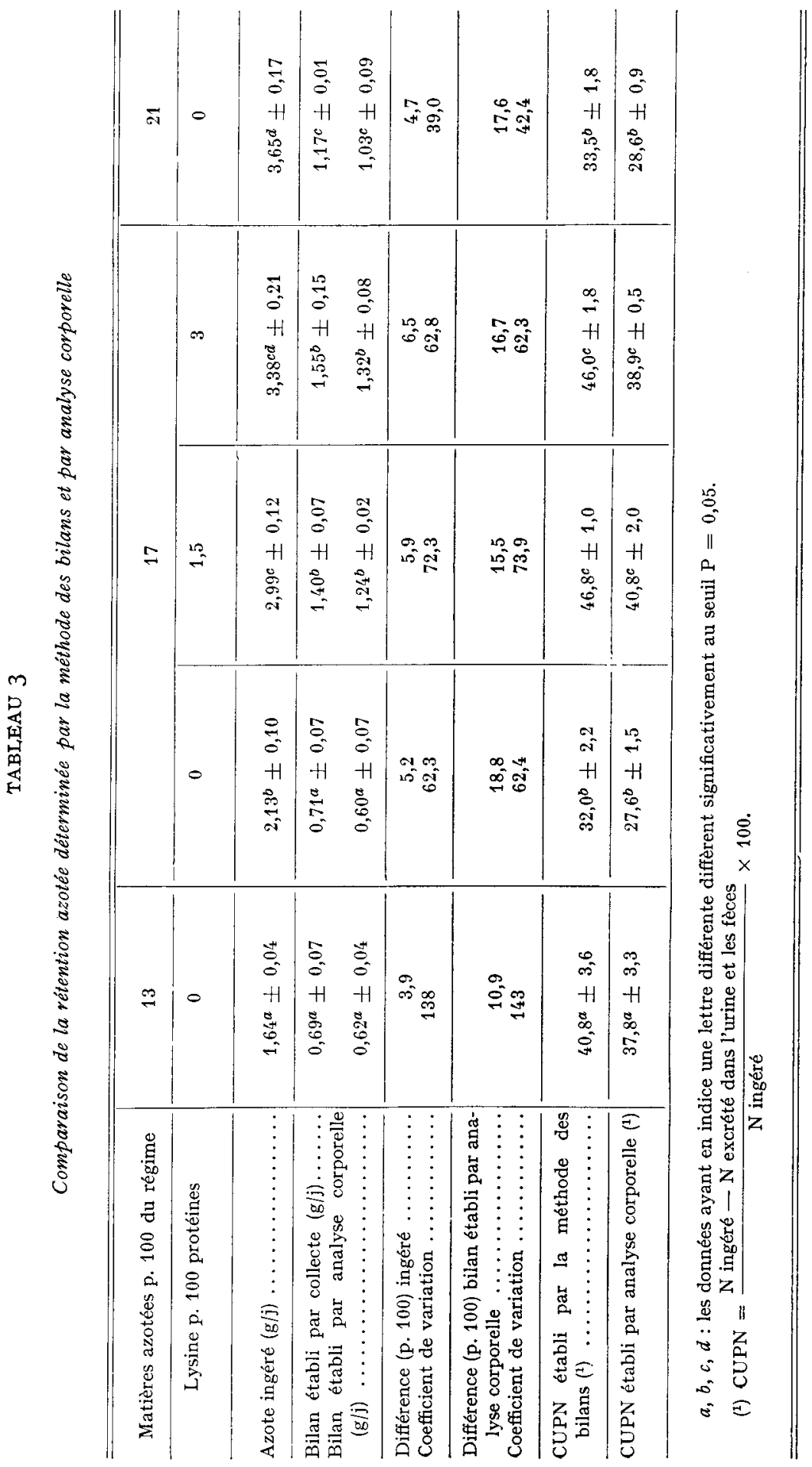




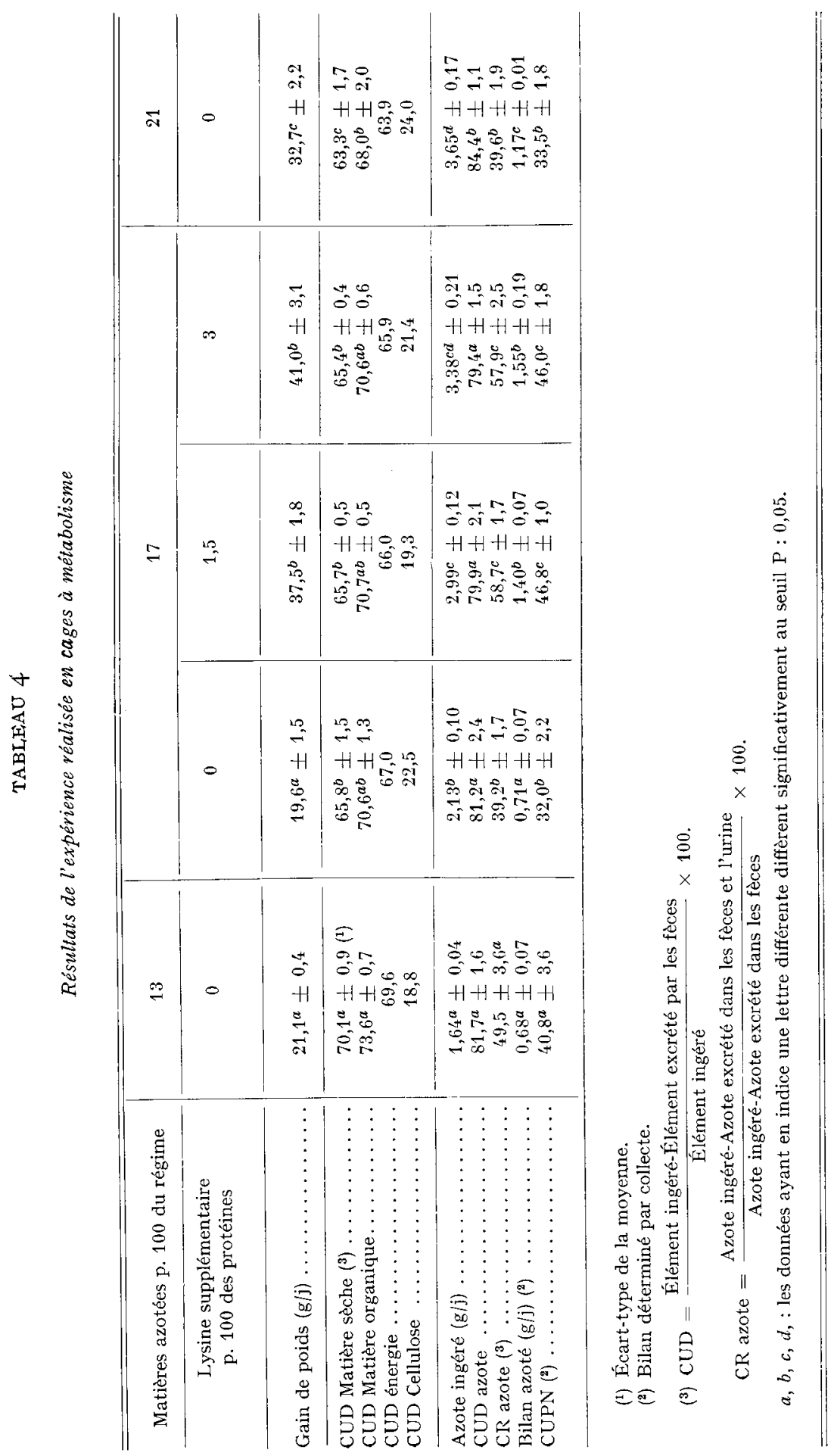




\section{C. - Bilan azoté et coefficient de rétention azotée}

Les bilans établis par collecte sont supérieurs de Io à $20 \mathrm{p}$. Ioo à ceux déterminés par analyse corporelle (tabl. 3). De plus, la différence relative entre ces deux types de bilan présente une variabilité considérable. Cependant, les différences moyennes entre les 2 valeurs du bilan ne représentent que 3,8 à 6,9 p. Ioo de l'azote ingéré.

En outre, malgré ces différences les bilans établis par les deux méthodes permettent de classer de façon analogue les 5 régimes étudiés.

La rétention azotée varie de façon parallèle au gain de poids (tab1. 4). Elle est fortement augmentée par l'élévation du taux protéique et la supplémentation en lysine. Le coefficient de rétention azotée est également amélioré dans les mêmes conditions.

\section{DISCUSSION}

\section{A. - Comparaison des deux méthodes d'estimation du bilan azoté}

Il semble tout d'abord utile de revenir sur l'écart enregistré entre le bilan azoté déterminé par collecte et celui établi par analyse corporelle. Cet écart peut paraître important mais en fait, il est du même ordre de grandeur que ceux enregistrés par la plupart des auteurs ayant réalisé cette comparaison sur d'autres espèces (tabl. 5). Seuls des auteurs travaillant dans des conditions très rigoureuses, difficilement compatibles avec un protocole expérimental comprenant plusieurs régimes, trouvent un bon accord entre les deux méthodes (BECKER et HARNisch, I958) ; cependant, le dispositif utilisé par ces auteurs ne permet plus de séparer lors de la récolte l'azote urinaire de l'azote fécal.

Ce décalage entre les 2 types de bilans est dû, d'après NIELSEN (I970) à une surestimation importante de l'azote retenu par la méthode des collectes. Cette surestimation s'explique par la difficulté à recueillir la totalité de l'azote urinaire et fécal et par l'existence d'autres pertes azotées notamment la chute des poils. Chez le Lapin en effet, l'excrétion d'azote urinaire est approximativement deux fois plus importante que l'excrétion fécale; or, l'urine, très visqueuse, colle aux parois de la cage et fermente. De plus, la fourrure abondante de cet animal implique une importante chute de poils. Les pertes d'azote se situent donc vraisemblablement en grande partie à ces deux niveaux.

Par ailleurs, notre mode de détermination de la teneur en azote des animaux au début de l'expérience est critiquable car il porte sur un petit nombre de lapins. La faible variabilité enregistrée sur ce critère laisse cependant penser que l'erreur ainsi commise reste peu importante. L'analyse corporelle a donc vraisemblablement donné, comme le pense NIELSEN (I970) une valeur du bilan plus juste que la méthode de collecte. En outre, la comparaison des bilans établis par les 2 méthodes permet d'aboutir à des conclusions identiques. C'est ainsi que les différences mises en évidence entre les bilans sont beaucoup plus élevées que l'écart enregistré entre 2 estimations du même bilan. Nos méthodes - malgré leurs imperfections - nous ont donc permis de travailler dans des conditions satisfaisantes, compte tenu des différences importantes de compositions et de performances entre les régimes. 
Enfin, il convient de revenir sur la signification exacte du bilan azoté tel que nous avons pu le déterminer. En effet, le contenu du tube digestif représente environ Io p. Ioo du poids vif du lapin (LEBAs, I969). Les bilans déterminés par les deux méthodes incluent donc en partie des variations de la quantité d'azote du contenu digestif. Ils ne rendent pas exclusivement compte de la synthèse protéique au niveau des tissus de l'animal. Seule l'analyse corporelle de lapins dont le tube digestif serait vidé au moment de l'abattage permettrait d'obtenir un tel résultat.

\section{TABLEAU 5}

Résultats de comparaison des 2 méthodes d'estimation du bilan azoté d'après différents auteurs

\begin{tabular}{|c|c|c|}
\hline Auteurs & Espèce ćtudiée & $\begin{array}{c}\text { Différence p. } 100 \\
\text { du bilan établi } \\
\text { par analyse corporelle }\end{array}$ \\
\hline Nehring et al. (1957) & $\begin{array}{l}\text { Rat } \\
\text { Porc }\end{array}$ & $\begin{array}{l}10 \text { à } 40 \\
13 \text { à } 23\end{array}$ \\
\hline BECKer et HaRnisch (1958) & Rat & 1 \\
\hline HARNISCH et BECKER (1958) & $\begin{array}{l}\text { Poulet } \\
\text { Porcelet }\end{array}$ & $\begin{array}{l}-1,6 \text { à } 2,2 \\
-0,3 \text { à }+0,5\end{array}$ \\
\hline $\begin{array}{l}\text { MULLER, WILMES } \\
\text { et KNAPPEN }(1960)\end{array}$ & Rat & $+4,1 \grave{a}-6,5$ \\
\hline RÉRAT (1961) & Rat & 27 à 40 \\
\hline BUTTERWORTH (1962) & Poulet & 16 \\
\hline Schiemann et al. (1962) & $\begin{array}{l}\text { Rat } \\
\text { Porc }\end{array}$ & $\begin{array}{l}10 \\
26,4\end{array}$ \\
\hline HENRY (1965) & Rat & 11 à 47 \\
\hline Davidson et WiLliams (1968) & Poulet & 15 \\
\hline NiELSEN $(1970)$ & Porc & 4,9 à 26 \\
\hline Fuller et BOYNE (1971) & Porc & 13 à 33 \\
\hline Présent travail (1974) & Lapin & 11 à 19 \\
\hline
\end{tabular}

\section{B. - Besoin en lysine du lapin}

Nos résultats montrent que l'augmentation du taux de matières azotées améliore les performances de croissance. Pour les régimes non supplémentés, nous trouvons des résultats proches de ceux de L,EBAs (1973) qui a situé le besoin en matières azotées à environ $20 \mathrm{p}$. roo du régime lorsque celles-ci sont apportées presque uniquement par le tourteau de sésame. Par contre, pour les régimes supplémentés, on n'observe un effet du taux protéique qu'entre ${ }_{3} 3$ et I 7 p. Ioo. Les différences entre les régimes 
à I7 p. IOO et $2 \mathrm{I}$ p. IOO de matières azotées non supplémentées sont donc dues à une déficience en lysine, et non en matières azotées, du régime à $\mathrm{I} 7 \mathrm{p}$. Ioo de matières azotées. On peut donc estimer qu'un régime à $\mathrm{I} 7 \mathrm{p}$. Ioo de matières azotées bien équilibrées couvre les besoins azotés du Lapin.

La supplémentation en lysine a amélioré les performances de croissance à tous les taux de matières azotées, confirmant les données de GaMan et Fisher (I97I) et de CHEFkE (I97I). L'ampleur de cette amélioration varie cependant avec le taux protéique. Elle est très forte à $\mathrm{I} 7 \mathrm{p}$. Ioo de matières azotées ( $48 \mathrm{p}$. Ioo pour le gain de poids), indiquant que la lysine est vraisemblablement le seul acide aminé déficient dans ce régime et que son apport permet d'obtenir des performances proches de l'optimum. Par contre, à I3 p. Ioo de matières azotées l'addition de 1ysine améliore moins les performances (34 p. Ioo pour la croissance), et celles-ci restent médiocres. Ces faits ne peuvent être impliqués à la seule lysine puisqu'on n'observe pas de différences entre les taux de I,5 et $3 \mathrm{p}$. Ioo de lysine supplémentaire.

Il doit donc exister des facteurs limitants secondaires, qui pourraient être par exemple les acides aminés soufrés. Le taux de ceux-ci s'élève à $0,5^{8} \mathrm{p}$. Ioo du régime, c'est-à-dire qu'il est inférieur à la zone où par ailleurs nous avons situé le besoin (Colin, ARkhurst et LEBAS, I973). Enfin, l'amélioration des performances lors de la supplémentation est faible à $2 \mathrm{I}$ p. Ioo de matières azotées $(9 \mathrm{p}$. Ioo) et les performances permises par le régime non supplémenté sont bonnes. Le déficit relatif en lysine est donc peu important à ce taux de matières azotées.

On peut d'après cette expérience, estimer un ordre de grandeur du besoin. Celui-ci se situerait dans une zone comprise entre 0,55 et $0,70 \mathrm{p}$. Ioo du régime, soit 3,5 à $4 \mathrm{p}$. Ioo des matières azotées. Il serait également, en l'exprimant en quantités ingérées par jour, de 500 à $600 \mathrm{mg}$. Il paraît cependant difficile d'avancer à partir de ces seules expériences une valeur précise du besoin en lysine du Lapin. En effet, nos résultats n'ont pas permis de mettre en évidence un taux de lysine à partir duquel l'addition de nouvelles doses de cet acide aminé au régime ne modifie plus les performances. Or le besoin au sens strict du terme est défini par ce taux (BRESSANI et MERTz, I958). Par ailleurs, une partie importante de la lysine ingérée est apportée par les protéines de sésame. Une fraction de cette lysine peut-être indisponible, ce qui entraînerait une légère surestimation de nos estimations. Enfin, comme l'ont montré HEwITH et LEWIS (I972) chez le Poulet, le besoin en lysine varie avec l'équilibre des autres acides aminés indispensables entre eux. Tous ces faits montrent bien qu'il serait hasardeux de vouloir avancer une valeur précise du besoin à partir d'un seul type de régimes.

L'ordre de grandeur du besoin tel qu'il est établi dans cette expérience est très proche de celui déterminé par ADAMSON et FiSHER (I973) à partir de mélanges d'acides aminés purifiés : o,70 p. Ioo du régime. Il est par contre nettement plus faible que celui avancé par CHEEKE (I97I) et que celui que nous avions nous-mêmes trouvé dans une expérience précédente (CoL,IN, r973).

Il convient en outre de remarquer que des taux élevés de lysine ne semblent présenter aucun effet dépressif sur la croissance des animaux, contrairement à ce qu'ont trouvé chez le Porc mâle castré Henry, Rérat et Tomassone, (I97I).

L'élévation du taux de matières azotées de la ration permet une meilleure utilisation digestive apparente des matières azotées, résultat déjà signalé chez le Lapin (Gl,Over et DuthiE, I958; SLADE et Robinson, I970) et chez d'autres espèces 
monogastriques, notamment le Porc (Henry, RÉRAt et Tomassone, I97I). Il est généralement attribué au fait que la contribution de la fraction azotée endogène est plus élevée au taux azoté le plus bas.

L'abaissement des coefficients d'utilisation digestive de la matière sèche, de la matière organique et de l'énergie lors de l'augmentation du taux d'incorporation du tourteau de sésame semble dû à une augmentation de la teneur en cellulose du régime. Une dépression de l'utilisation digestive de la ration lors d'une élévation de son taux de cellulose est en effet un phénomène connu chez le Lapin (BEsEdina et PEREL'DIK, I970). La cellulose elle-même semble mieux digérée lorsque son taux dans la ration augmente. Un tel phénomène a déjà été signalé par AGUILERA (I973), dans des conditions identiques. Cependant, il convient de rester prudent dans l'interprétation de cette évolution car les coefficients d'utilisation digestive de la cellulose ayant été établis à partir d'échantillons moyens, nous ne possédons aucune information sur la variabilité. L'addition de lysine au régime ne modifie pas l'utilisation digestive apparente des différents éléments de la ration, comme l'ont constaté chez le Porc, RÉRat et Lougnon (1965) et RÉRAT et al. (I970).

Le coefficient de rétention azotée est abaissé lorsque le taux protéique croit, et amélioré par la supplémentation en lysine. L'augmentation des performances de croissances des animaux lors de la supplémentation s'explique en partie par cette meilleure utilisation métabolique des protéines. Tous ces résultats sont à rapprocher de ceux établis chez le Porc (RÉRAT et HEnRY, I964 ; RÉRAT et al., I970) et le Rat (HENRY et RÉRAT, Ig66).

Ce travail montre un besoin en lysine du Lapin comparable par son ordre de grandeur à celui du Rat (RÉRAT et HENRy, I963) ou du Porc mâle castré (Henry, RÉRA'T et Tomassone, I97I). Ce fait est à souligner car les particularités digestives du Lapin pourraient au contraire laisser penser à une relative autonomie de cet animal par rapport à la teneur en lysine de sa ration. En effet, les protéines du contenu cæcal sont en majorité des protéines bactériennes riches en lysine (MARTY et CARLES, I968; MARTY, I97I). La lysine est abondante dans les cæcotrophes (FERRANDO et al., I970; Yoshida et al., I97I). En outre DJOUkHam (I973), a montré que la teneur en lysine des cæcotrophes paraît peu affectée par la nature des protéines alimentaires. La cæcotrophie et la synthèse d'acides aminés essentiels par les Bactéries cæcales pourraient donc apparaître comme une autosupplémentation en lysine du Lapin. Celle-ci ne semble cependant présenter qu'un intérêt très limité pour l'animal, puisque ce dernier est incapable d'utiliser dans de bonnes conditions des régimes pauvres en lysine.

\section{CONCLUSION}

En conclusion, on peut affirmer que le Lapin est sensible à la teneur en lysine des matières azotées de sa ration. L'addition de lysine à un régime déficient améliore à la fois le niveau d'ingestion de l'aliment et l'utilisation métabolique de ses protéines. Pour un régime à $I 7$ p. Ioo de protéine de sésame, le besoin en lysine de Lapin se situe aux environs de 0,55 à 0,70 p. Ioo du régime. 


\section{SUMMARY}

\section{LYSINE SUPPLEMENTATION OF SESAME OIL-MEAI, DIETS IN THE RABBIT. EFFECTS ON GROW'TH PERFORMANCES AND NITROGEN BALANCE ESTIMATED BY MEANS OF TWO METHODS}

The effects of L-lysine supplementation of diets containing sesame oil-meal were studied according to a factorial scheme including 3 crude protein levels ( $13-17-2$ I . Ioo) and 3 doses of supplementary lysine (O-I.5-3 p. Ioo of the crude protein).

Lysine supplementation allowed improvement of growth performances. The effects were particularly marked at a crude protein level of $I_{7}$ p. IOo, less pronounced at $I_{3}$ p. Ioo with poor weight gains and low when the level was 2 I p. Ioo.

These results indicate that the crude protein and lysine requirements range about I 7 p. Ioo and 0.65 p. Ioo, respectively, of the diet.

Furthermore, 5 of the previous diets ( 3 non supplemented diets and 2 diets at 17 p. Ioo supplemented crude protein) were given to animals kept in metabolism crates and the studies showed that :

- the nitrogen balance varies parallel to the weight gain

- elevation of the crude protein level reduces the nitrogen retention coefficient and improves the apparent digestibility of nitrogen

- L-lysine supplementation increases the nitrogen retention coefficient and does not change the apparent digestibility of crude protein.

In addition, during this experiment we compared nitrogen balances determined by collectings with those established by body analyses. The difference recorded (from I I to I9 p. IOO) is similar to those reported in the literature for other species. However, the 2 types of balances allow identical classification of the diets.

\section{RÉFÉRENCES BIBLIOGRAPHIQUES}

Adamson I., Fisher H., I973. The amino acid requirement of the growing Rabbit : an estimate of quantitative needs. $J$. Nutr., 103, I306-1 3 Io.

Aguilera J. F., I973. The infuence of the dietary protein level on the digestibility, nutritive value and nitrogen balance in growing rabbits. Ve congrès international de Cuniculiculture, sept. 1973 . Côme (Italie).

Becker M., Harnisch S., 1958. Neue Untersuchungen über die Gültigkeit und Fxagktheit von Stickstoffbilanzen bei Stoffwechselversuchen an lebenden Tieren. Arch. Tierernähr., 8, 40I-4I9.

Besedina G. G., Perel'dik N. S., i97o. Proportion of fibre in the diet and digestibility of feeds, Krolik. Zver, 6, 3-5.

Bressani R., Mertz E. T., I958. Relationship of protein level to the minimum lysine requirement of the Rat. J. Nutr., 65, $48 \mathrm{I}-49 \mathrm{I}$.

Butterworth M. H., I962. Evaluation of cereal protein quality for Chicks by three different biological techniques. J. Sci. Food. Agric., 13, 6-I5.

Cheеке P. R., I97x. Arginine, lysine and methionine needs of the growing rabbit. Nutr. Repts Int., 3, $123-128$.

Colın M., I973. Influence de l'addition de lysine à des régines à base de tourteau de sésame chez le Lapin. Journées Recherches Avicole et Cunicole, I9-21, I.T.A.V.I. ed.

Colin M., Arkhurst G., Lebas F., I973. Effets de l'addition de méthionine au régime alimentaire sur les performances de croissance du Lapin. Ann. Zootech, 22 485-49I.

Davidson J., Williams R. B., I968. A note on the estimation of nitrogen retention in Chicks by body analysis and balance methods. Brit J. Nutr., 22, $29 \mathrm{I}-295$.

Djoukam J., 1973. Coecotrophie et nutrition azotée du Lapin. Thèse Doc. $3^{\mathrm{e}}$ cycle, Université Paris VI.

Ferrando R., Wolter R., Vitat J.-C., Megard J.-P., r97o. Teneur en acides aminés des deux catégories de fèces du Lapin : coecotrophes et fèces dures. C. R. Acad. Sci. Paris, 270, série D, 22022204 . 
Fuller M. F., Boyne A. W., 1971. The effects of environmental temperature on the growth and metabolism of Pigs given different amount of food. Brit J. Nutr., 25, 259-272.

Gaman E., Fisher IT., I97I. The essentiality of arginine, lysine and methionine for the growing Rabbit. Nutr. Repts. Int., 1, 57-64.

Glover J., Duthie D. W., I958. The apparent digestibility of crude protein by non Ruminants and Ruminants. J. Agric. Sci., 51, 289-293.

HARnich S., Becker M., I958. Neue Untersuchungen über die Gültigkeit und Exagktheit von Stickstoffbilanzen bei Stoffwechselversuchen an lebenden Tieren. Arch. Tierernähr., 8, 430-432.

HENRY K. M., I965. A comparison of biological methods with Rats for determining the nutritive value of proteins. Brit. J. Nutr, 19, I25-1 35 .

Henry Y., Rérat A., I966. In Jacouot R. et Rérat A. La valeur biologique des protéines. Ier Congrès Mondial d'Alimentation animale (Madrid) 1, 329.407.

Henry Y., Rérat A., Tomassone R., ig7r. Étude du besoin en lysine du Porc en croissance finition. Application de l'analyse multidimensionnelle. Ann. Zootech., 20, 521-550.

Hewits D., Lewis D., I972. The amino acid requirement of the growing Chick. I. Determination of amino acid requirements. Br. Poultry. Sci., 13, $449-464$.

Lebas F., 1969. L'alimentation du Lapin. Bull. Soc. Sci. Hyg. Aliment, 57, 245-268.

Lebas F., 1973. Effet de la teneur en protéines de ration à base de soja ou de sésame sur les performances de croissance du Lapin. Ann. Zootech., 22, 83-92.

Marty J., I971. Origine et intérêt nutritionnel des acides aminês et des acides organiques dans le caecum du Lapin. Thèse de doctorat d'état, Université de Toulouse.

Marty J., Carles J., 1968. Les acides aminés dans le cæcum du Lapin. C. R. Acad. Sci. Paris, 267, série D, 638-64I.

Müller R., Wilmes G., Knappen F., I96o. Beiträge zur biologischen Proteinbewertung. Arch. Tievernähr., 10, 326-345.

Nehring K., Laube W., Schwerdtfeger E., Schiemann R., Haesler E., Hoffmann T., 1957. Die Bestimmung der biologischen Wertigkeit des Eiweisses. N. Bilanz und N-Ansatz. Biochem Ztschr, 328, 549-56r.

Nielsen J., I97o. The energie value of balanced rations for growing Pigs by different methods. Beretn. Forsogslab. Kobenhavn, $\mathrm{n}^{0} 38 \mathrm{I}$.

Rérat A., Ig6I. Les antibiotiques dans l'alimentation animale. Ann. Nutr. Alim., 15, 75-165.

Rérat A., Henry Y., I963. Besoin en lysine du Rat en croissance : principe d'une méthodologie et résultats expérimentaux. C. R. Ac. Sci., 25', 3045-3048.

Rérat A., Henry Y., I964. Étude du besoin azoté chez le Porc en croissance. 1 . Utilisation de la farine de poisson à 3 taux différents. Ann. Zootech., 13, 5-34.

RÉrat A., Lougnon J., I965. Supplémentation par la L-lysine d'un régime végétal simplifié chez le Porc en croissance. Ann. Zootech., 14, 247-260.

Rerat A., Lerner J., Henry Y., Bourdon D., 197o. Étude du besoin en lysine du Porc en croissance en relation avec le taux énergétique du régime. Journées Rech. Porcine en France, 79-84, I. N. R. A I. T. P. éd., Paris.

Schiemann R., Jentsch W., Klippel W., Schmidt F., Trela S., Tscheschmedschiew B., I962. Vergleichende Untersuchungen zwischen der Methodik der Gesamtstoffwechselmessungen und der Tierkörper-analytik an wachsenden Ratten und Schweinen. Arch. Tierernähr., 12, 32I-342.

Slade L. M., Robinson D. W., I97o. Nitrogen metabolism in non Ruminant herbivore. II. Comparative aspect of protein digestion. J. Anim Sci., 30, 76I-763.

Yoshida T., Pleasants J. R., Reddy B. S., Wostmann B. S., I97I. Amino acid composition of cecal contents and feces in germfree and conventional Rabbits. J. Nutr., 101, 1423-I430. 